EOMmun Communication et organisation

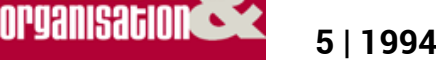

La communication interne : une approche croisée

\title{
Un état des lieux en guise d'éditorial
}

\section{Hugues Hotier}

\section{OpenEdition}

Journals

Édition électronique

URL : http://journals.openedition.org/communicationorganisation/1693

DOI : 10.4000/communicationorganisation. 1693

ISSN : $1775-3546$

\section{Éditeur}

Presses universitaires de Bordeaux

Édition imprimée

Date de publication : 1 mai 1994

ISSN : $1168-5549$

\section{Référence électronique}

Hugues Hotier, «Un état des lieux en guise d'éditorial », Communication et organisation [En ligne], 5 |

1994, mis en ligne le 26 mars 2012, consulté le 21 septembre 2020. URL : http://

journals.openedition.org/communicationorganisation/1693; DOI : https://doi.org/10.4000/

communicationorganisation. 1693

Ce document a été généré automatiquement le 21 septembre 2020.

(c) Presses universitaires de Bordeaux 


\title{
Un état des lieux en guise d'éditorial
}

\author{
Hugues Hotier
}

1 Avec ce numéro, le cinquième, Communication \& Organisation entre dans sa troisième année d'existence. C'est-à-dire que cette revue donne des gages de pérennité et qu'elle fidélise un lectorat qui s'habitue aux problématiques qu'elle pose. A leur traitement aussi, alliant les hypothèses et les analyses des chercheurs aux témoignages et aux réflexions des praticiens.

2 Autre façon de s'inscrire dans la durée, la thématique des dossiers de nos prochains numéros est établie pour les deux années à venir. Une thématique diversifiée et qui ne néglige aucun des aspects de la communication des organisations; le dossier du numéro 6 traitera de la communication locale et celui du numéro 7 sera consacré à la communication sur l'histoire de l'organisation. Le premier (novembre 94) sera coordonné par Bernard Dagenais, de l'Université Laval de Québec tandis que le second (mai 1995) est pris en charge par deux enseignants-chercheurs bordelais, exerçant tous deux en IUT: Dominique Blin (Université Michel de Montaigne) et Gino Gramacia (Université Bordeaux I, à dominante scientifique et juridique).

3 Mais les dossiers thématiques ne sont pas tout, même s'ils sont importants puisque leur est réservée la moitié de chaque volume, soit entre 125 et 150 pages. Nous tenons à ce que Communication \& Organisation soit un lieu de publication toujours ouvert et disponible. C'est pourquoi existent des rubriques qui peuvent accueillir des articles hors thème et permettent ainsi aux auteurs, de plus en plus nombreux à nous proposer des textes, d'être publiés sans trop attendre. Pour peu, bien entendu que ces textes aient été acceptés par les membres du comité de lecture qui, en France et dans de nombreux pays de langue française, acceptent de nous aider selon les procédures en usage dans les revues de la communauté scientifique internationale. Dans une prochaine livraison, nous publierons les noms de ces personnalités reconnues qui acceptent de nous consacrer une part de leur temps et nous apportent le secours de leur compétence et de leur vigilance.

4 Comme vous pouvez le constater, nous recourons désormais à des techniques graphiques qui nous permettent d'offrir une meilleure présentation et une meilleure qualité de lecture. La maquette a évolué quelque peu grâce à Christine Pouillet, qui a en 
charge la saisie et mise en page, et aux conseils avisés de Philippe Loquay, membre du Groupe de Recherche en Communication des Organisation (GREC/O) qui édite cette revue.

5 Puisqu'on en parle, nous n'hésiterons pas à évoquer l'activité importante du GREC/O qui vient de réunir des chercheurs et des personnalités du monde hospitalier pour un colloque, coordonné par Valérie Carayol, sur «Communication et Hopital». Les actes seront publiés à la fin de cette année par notre revue sous la forme d'un numéro hors série qu'on peut commander dès à présent. Le GREC/O travaille actuellement dans deux directions. D'une part, il poursuit sa réflexion théorique sur l'induction dans la communication. Les seize chercheurs qui le composent travaillent sur ce sujet commun et il est fait appel, quand besoin est, à des personnalités extérieures car l'approche pluridisciplinaire est évidemment indispensable ici. D’autre part, un groupe formé de membres du GREC/O et de chercheurs du Département de communication de la Faculté de Lettres et des Sciences Humaines Ben M'Sik de Casablanca (Université Hassan II de Mohammedia) mène une étude sur "Communication institutionnelle et cultures: approche comparative France-Maroc » qui donnera lieu à un colloque à Bordeaux et à Casablanca en mai 1995. Là aussi, un numéro hors série de notre revue permettra de donner aux actes la diffusion la plus large possible.

6 Mais pour le moment, nous vous proposons un numéro construit autour d'un dossier consacré à un sujet de la plus grande importance : la communication interne. Ce dossier a été élaboré et coordonné par Béatrice Galinon-Mélénec, maître de conférences à l'Université Michel de Montaigne de Bordeaux 3 où elle assume la responsabilité pédagogique de l'option «communication interne» du DESS en communication des organisations. Elle a publié, aux Editions d'organisation, un ouvrage remarqué sur le projet d'entreprise appliqué à l'université. C'est-à-dire que nous avons fait appel à une compétence reconnue qui a su s'entourer des meilleurs spécialistes ainsi que vous pourrez le constater.

7 J'aimerais terminer sur une double conclusion; s'il est vrai que le nombre des auteurs qui nous proposent des articles croît aussi vite que celui de nos abonnés, il n'est pas moins vrai que nous avons besoin d'étendre et de diversifier le réseau de nos collaborateurs tout comme nous souhaitons, évidemment augmenter et hétérogénéiser le fichier de nos abonnés. En d'autres termes, cette conclusion est une ouverture... 